\title{
INFLUENCIA DE SOFTWARE EDUCATIVO EN LA CONSOLIDACIÓN DEL SISTEMA DE NUMERACIÓN POSICIONAL
}

\author{
THE INFLUENCE OF AN EDUCATIONAL SOFTWARE IN THE \\ CONSOLIDATION OF POSITIONAL NUMERAL SYSTEM
}

\begin{abstract}
Efraín A. Hoyos Salcedo ${ }^{2}$, Leonardo D. Restrepo Alape
${ }^{1}$ Departamento de Matemáticas, Universidad del Quindío, Colombia

2 Departamento de Matemáticas, Universidad del Quindío, Colombia

${ }^{3}$ Universidad del Tolima, Resultados de investigación del proyecto influencia de un software educativo, en la consolida ción del sistema decimal de numeración a partir de la lógica de construcción de sistemas posicionales, Universidad del Quindío, 2014-2015

Recibido: Mayo 5 de 2015 Aceptado: 3 de Agosto de 2015

*Correspondencia del autor: E-mail: cminoli@uniquindio.edu.co
\end{abstract}

\begin{abstract}
RESUMEN
Este trabajo presenta una propuesta de enseñanza apoyada en material didáctico manipulativo y computarizado para la consolidación del sistema de numeración posicional en base diez a partir de la lógica de los sistemas posicionales. Se estableció experimental y cualitativamente los efectos del uso de la propuesta en la consolidación conceptual del sistema de numeración posicional. Es de resaltar el diseño y desarrollo de un software educativo por parte de los investigadores, considerando una serie de elementos didácticos y teóricos para facilitar en principio, la consolidación de los sistemas de numeración posicional. El estudio permitió evidenciar que una metodología de enseñanza enriquecida con materiales manipulativos y computarizados se presenta como una importante herramienta didáctica en la enseñanza y en la consolidación de los sistemas posicionales de numeración.
\end{abstract}

Palabras Clave: Software educativo, sistema de numeración posicional, consolidación del aprendizaje.

\begin{abstract}
This paper presents a methodological proposal based on a manipulative and computerized didactic material to consolidate the positional numeral system in base ten from the positional systems logic. It was established experimentally and qualitatively the effects of the proposal in the conceptual strengthening of positional numeral system. It is important to highlight the design and development of the educational software by the researches, considering a series of didactic and theoretical elements to facilitate the positional numeral system consolidation. This study evidenced that an enriching methodology based on manipulative and computerized materials is an important didactic tool in teaching and in the consolidation of positional numeral systems.
\end{abstract}

Keywords: Educational software, positional numeral system, learning consolidation. 


\section{INTRODUCCIÓN}

Los sistemas de numeración son de gran importancia en el estudio de las matemáticas, no solamente por su contexto histórico sino como herramienta de representación y de cálculo en la resolución de problemas. $\mathrm{Su}$ enseñanza y aprendizaje son fundamentales para la comprensión del concepto de número, es por eso que forman parte del núcleo curricular de los programas de matemáticas en educación preescolar y de básica primaria.

Desde una perspectiva moderna, los sistemas de numeración se pueden clasificar en sistemas aditivos, sistemas multiplicativos y sistemas posicionales. En un sistema aditivo, se definen símbolos para la unidad, la base y las potencias de la base, de esta forma el número representado se obtiene por medio de la adición de los símbolos que componen la representación. Por su parte, en los sistemas multiplicativos se definen símbolos para la unidad, la base, las potencias de la base y todos los números comprendidos entre la unidad y la base, de esta forma el número representado se obtiene multiplicando cada potencia de la base por el valor del símbolo que le precede y sumando los resultados junto con las unidades. Y finalmente en los sistemas posicionales, se definen símbolos para la unidad y los números comprendidos entre la unidad y la base, una característica importante de estos sistemas es que también se define un símbolo denominado cero para explicar la ausencia de unidades, de esta forma tanto la base como las potencias de la base se representan como combinación entre la unidad y el cero, (Godino,2003).

En general los sistemas de numeración posicional como el hindú-arábigo se construyen bajo las siguientes reglas, ver (Knuth,1981): primero se elige un número denominado base $b$, el cual determina el número de símbolos usados en el sistema, dichos símbolos son denominados dígitos $\{0, \ldots, b-1\}$. Cada $b$ unidades de un orden forman una unidad del orden siguiente, esto se expresa de forma polinomial. La falta de unidades en un orden del lado derecho se expresa por medio de un cero. Entonces, todo número natural $\mathrm{N}$ se puede expresar de manera única mediante el siguiente polinomio:

$\mathrm{N}=\left(\mathrm{a}_{k} \mathrm{a}_{k-1} \ldots \mathrm{a}_{1} \mathrm{a}_{0}\right)_{\mathrm{b}}=\mathrm{a}_{\mathrm{k}} \cdot \mathrm{b}^{\mathrm{k}}+\mathrm{a}_{\mathrm{k}-1} \cdot \mathrm{b}^{\mathrm{k}-1} \ldots+\mathrm{a}_{1} \cdot \mathrm{b}^{1}+\mathrm{a}_{0}$

donde $a_{k}<b$. Entre los sistemas de numeración posicional se destaca el sistema en base diez $(b=10)$, ya que su relativo bajo número de símbolos (asociado a los dedos de las manos) lo hace eficiente para realizar cálculos mientras permite representar números grandes con facilidad.

Con respecto a la enseñanza y al aprendizaje de los sistemas de numeración, estos se introducen en el sistema educativo como parte integral y fundamental del aprendizaje de las matemáticas. Para usar efectivamente un sistema notacional se requiere entender la relación entre el simbolismo y los conceptos representados, y del conocimiento de las reglas para manipular dicha notación (Lengnink, 2010), según Jorge Castaño et. al. (Castaño,1990), el estudio de los sistemas de numeración posicional exige del niño el manejo operatorio de la composición y descomposición aditiva y del manejo del esquema multiplicativo, este último se refleja en el hecho de poder operar de forma simultánea con unidades de diferentes ordenes y de establecer la equivalencia de varias unidades de un orden con relación a las de un orden inmediatamente inferior.

Para comprender progresivamente el sistema de numeración posicional, los estándares curriculares de Colombia proponen el desarrollo de las siguientes habilidades en los grados tercero y quinto: usar representaciones principalmente concretas y pictóricas para realizar equivalencias de un número en las diferentes unidades del sistema decimal, justificar el valor de posición en el sistema de numeración decimal en relación con el conteo recurrente de unidades, ver (Ramírez,2011), (Lengnink,2010), (MEN,2003).

En relación con el uso de materiales didácticos para el estudio de los sistemas de numeración Godino y colaboradores en su texto sistemas numéricos y su didáctica para maestros (Godino,2003) clasifican los materiales didácticos para el aprendizaje del sistema de numeración posicional en modelos proporcionales de base 10, en los cuales la decena se expresa diez veces mayor en tamaño que la unidad, como por ejemplo los bloques multibase, grupos de palillos, etc. Y los modelos no proporcionales donde las diferencias del valor posicional se indican por medio de un color, una posición o una regla a definir, por ejemplo: los ábacos, el dinero, etc. Adicionalmente, hace una lista de los materiales didácticos utilizados con más frecuencia en las aulas de clase. Entre ellos se destaca el ábaco, los bloques multibase y las regletas de Cuisinare. Por otra parte, la tecnología computacional juega un 
papel importante como apoyo para el aprendizaje. Diversas investigaciones corroboran los efectos positivos que la tecnología tiene sobre el aprendizaje (Garay,2008). A nivel nacional, el Ministerio de Educación Colombiano es consciente de las posibilidades que brinda la informática y el uso de la nuevas tecnologías para la enseñanza de la matemáticas en general, según se afirma en el libro de los lineamientos curriculares de las matemáticas: "Las nuevas tecnologías amplían el campo de indagación sobre el cual actúan las estructuras cognitivas que se tiene, enriquecen el currículo con los nuevos paradigmas asociadas y los llevan a evolucionar" (MEN, 1999, 2003) . De otro lado, el grupo GEDES de la universidad del Quindío desde 1996 ha venido realizando una serie de investigaciones y ha desarrollado una diversidad de recursos educativos digitales en pro de la incorporación de las nuevas tecnologías como recursos didácticos para la enseñanza de las matemáticas. Es el caso de los proyectos Estudio experimental del uso del geoplano computarizado en la enseñanza de la geometría euclidiana (Hoyos,2002), Estrategia de intervención pedagógica con juegos computarizados que contribuyan a la consolidación del esquema multiplicativo (Hoyos,2007) y Representación de objetos tridimensionales utilizando multicubos (Hoyos, 2012). Con respecto a la enseñanza de los sistemas posicionales con tecnología informática se evidencia el trabajo de Cadavid (Cadavid,2013) quien a través de una plataforma denominada LMS (Learning managment systems) desarrolló una metodología para mejorar la comprensión del concepto de valor posicional en el sistema de numeración decimal. En la actualidad, se pueden encontrar recursos didácticos para la enseñanza del sistema decimal que pueden ser utilizados hasta en dispositivos móviles y tabletas, ver (Ventura,2011). Con respecto a investigaciones sobre la enseñanza del valor posicional en el sistema decimal, basado en la metodología resolución de situaciones problema asociado a las TIC, se encuentra el trabajo de Claudia Y. Salazar \& Yuly A. Vivas (Salazar C. y Vivas Y., 2013). Finalmente, en una investigación sobre el uso de software educativo y la educación artística a través de la experiencia de aula, permitiendo crear unidades didácticas con la finalidad de un aprendizaje significativo del sistema de numeración decimal (Guarín S. 2012), se afirma en relación con las experiencias de aula con TIC:
La experiencia de aula basada en el arte y las tics, para la enseñanza del sistema de numeración decimal, me ha posibilitado tener un acercamiento a las características individuales de los estudiantes, respetar y valorar los ritmos individuales que presentan para acercarse al conocimiento y, sobre todo resignificarme como maestra, ya que debe existir un equilibrio entre las normas, las teorías, la cultura y los códigos sociales. Pero sobre todo conservar la esperanza y la alegría que se siente al estar en una aula de clase.

Sin embargo, a pesar de los avances referenciados en el párrafo anterior aún es necesario el desarrollo de innovaciones educativas basadas en el uso de la informática identificando el papel del docente en este uso y canalizando su potencial mediante una capacitación que permita un mejoramiento educativo en los estudiantes, en particular, en el desarrollo del razonamiento numérico apoyado con software educativo. Aún no se evidencia el desarrollo de materiales didácticos o de estrategias para la enseñanza del sistema posicional en relación con bases diferentes a la base diez.

Las consideraciones anteriores nos conducen a la formulación de la siguiente pregunta: ¿En el caso de que el método complementado con juegos didácticos pueda influir significativamente en la consolidación del sistema decimal de numeración existen diferencias significativas entre las condiciones experimentales?

El presente artículo tiene por objeto presentar los resultados de una investigación en relación con el uso de una propuesta didáctica apoyada con material didáctico computarizado para la consolidación del sistema de numeración posicional en base 10 a partir de la lógica de los sistemas posicionales, particularmente, estamos interesados en establecer experimental y cualitativamente si esta metodología influye de forma significativa en el proceso de consolidación conceptual del sistema de numeración posicional en estudiantes de grado séptimo de básica secundaria de cuatro instituciones educativas del departamento del Quindío. El contenido de este artículo se presentará en cinco secciones: en la sección 2 se describen tanto la propuesta didáctica como los materiales educativos computarizados desarrollados por el grupo de investigación. Posteriormente en la sección 3 se describe la metodología de investigación utilizada. Luego, en la sección 4 se hace una discusión desde el punto de vista cuantitativo y cualitativo de los resultados. Finalmente, en la sección 5 se presentan las conclusiones del trabajo.

\section{PROPUESTA DIDÁCTICA}

La propuesta didáctica se basa en la teoría cognitiva 
del aprendizaje (Baroody,2000), se enfoca en el trabajo constructivista y colaborativo, esto es, el estudiante mediante el uso del software educativo y de las actividades desarrolladas por el grupo de investigadores tendrá la posibilidad de extrapolar, discutir y conjeturar sobre las relaciones matemáticas y las diferentes representaciones de los objetos que construye. Dicha propuesta en el aula se compone de tres fases las cuales se presentan a continuación:

- Estados previos (indagación). Mediante un diálogo con los estudiantes, el maestro relaciona el tema de la actividad anterior con el tema correspondiente a desarrollar. En esta etapa el maestro también indaga sobre los conocimientos previos que el estudiante tiene acerca del nuevo tema, y llama la atención sobre lo pertinente al nuevo lenguaje dentro de este.

- Desarrollo y descubrimiento (Orientación dirigida y libre). En ésta parte, el estudiante emplea las guías y el software desarrollado, el aprendizaje se realiza en grupos (normalmente dos estudiantes), lo que les permite intercambiar argumentos y llegar a los primeros acuerdos conceptuales con respecto al tema de estudio.

- Puesta en común. Esta etapa es de suma importancia en el proceso de aprendizaje. Aquí los estudiantes relatan las experiencias obtenidas en el desarrollo de la etapa anterior. Los estudiantes mediante acuerdos orientados por el maestro, forman un sistema de relaciones del objeto de estudio, lo cual los lleva a un nuevo nivel de aprendizaje. La intervención del maestro en ésta fase consiste en proporcionar a los estudiantes algunos panoramas generales de aquello que ellos ya conocen.

La propuesta se enfoca en promover la construcción de las razones que subyacen en el funcionamiento de los números por parte de los estudiantes. Se espera que lleguen a comprender los principios que rigen el sistema y las operaciones asociadas a la notación numérica. Las ideas centrales del desarrollo de esta propuesta se basan en los supuestos teóricos propuestos por F Terigi, Lerner y sus respectivos colaboradores, (Terigi,2007,Lerner,1994).
En cuanto a los temas a desarrollar, estos se eligieron en función de los saberes necesarios para lograr desarrollar la lógica del sistema posicional como lo son: conversión entre unidades de una misma base, representación de un número en diferentes bases, sumas y restas en base $\mathrm{n}$ y resolución de problemas de empaquetamiento.

Para las actividades en el aula se utilizaron dos programas de software educativo en el sistema operativo Windows, elaborados por el grupo de investigación y siguiendo los lineamientos de desarrollo de software educativo propuestos por Galvis (Galvis,1994). Este software se pueden descargar de forma gratuita en la siguiente dirección: http://academia.uniquindio.edu. co/academia/investigacion/gedes/index.php/descargas. El primero se denomina Casa de cambio, consiste de un conjunto de fichas de colores blanco, rosadas, azules y amarillas a las cuales se les asigna un valor según el color, por ejemplo, dos fichas blancas equivalen a una rosada, dos rosadas equivalen a una azul y dos azules equivalen a una amarilla. Para el desarrollo del juego en el computador los jugadores lanzan un dado virtual y el computador en su papel de banco entrega al jugador el número de fichas blancas que indique el dado, finalmente el Jugador deberá hacer las conversiones a fichas de otros colores de acuerdo a las equivalencias establecidas inicialmente. En el desarrollo del juego se pueden presentar situaciones en las que al estudiante se le lleve a realizar sumas y restas en diferentes bases, además de realizar el proceso inverso de conversión de base diez (10) a otras bases. La interfaz del juego se presenta en la Figura.1.

El segundo software educativo utilizado se denomina Ábaco de Arbey, su interfaz construida de forma tridimensional modela un ábaco abierto no convencional, pues está diseñado a manera de contenedor con diferentes secciones, las cuales permiten cambiar el número de elementos que se agrupan en cada orden (base). Dichas secciones están asignadas a las fichas de colores trabajadas en la casa de cambio, de esta forma el ábaco abierto en el computador se convierte en una herramienta visual complementaria a la casa de cambio, ya que permite al estudiante estructurar la lógica del juego y llevarla a una representación formal. La interfaz del software Ábaco de Arbey se presenta en la Figura 2. 
Influencia de software educativo en la consolidación del sistema de numeración. Acosta et al.
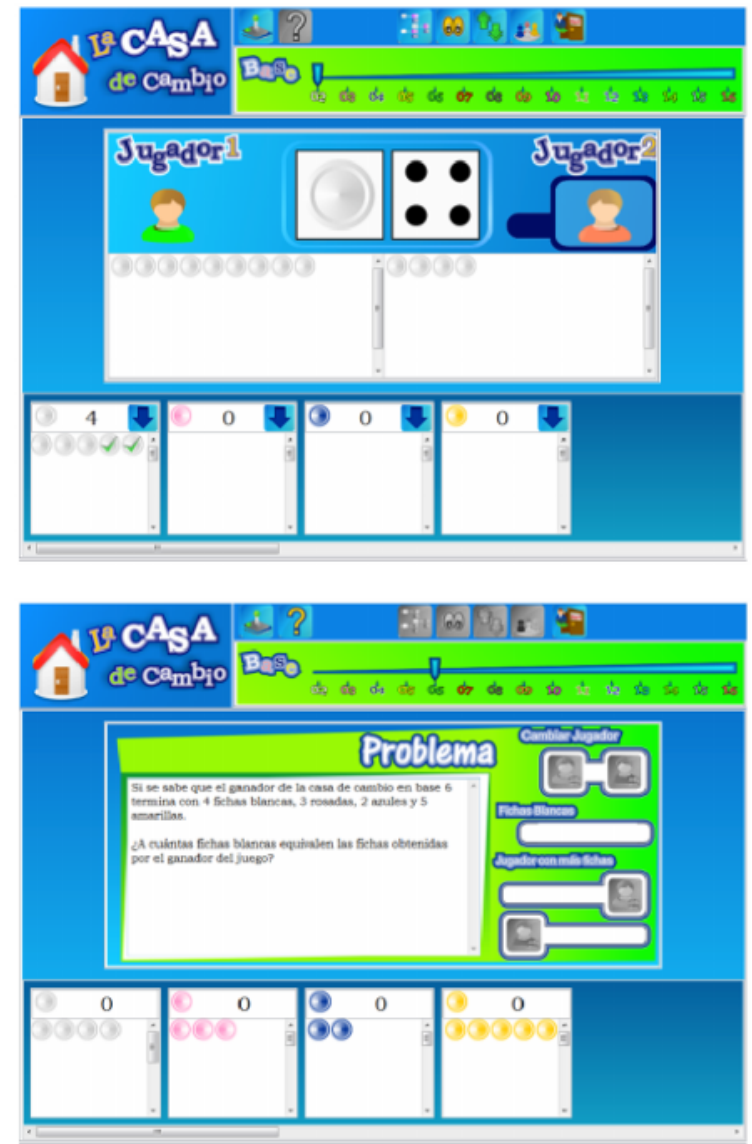

Figura 1. Interfaz Software Educativo la Casa de Cambio.

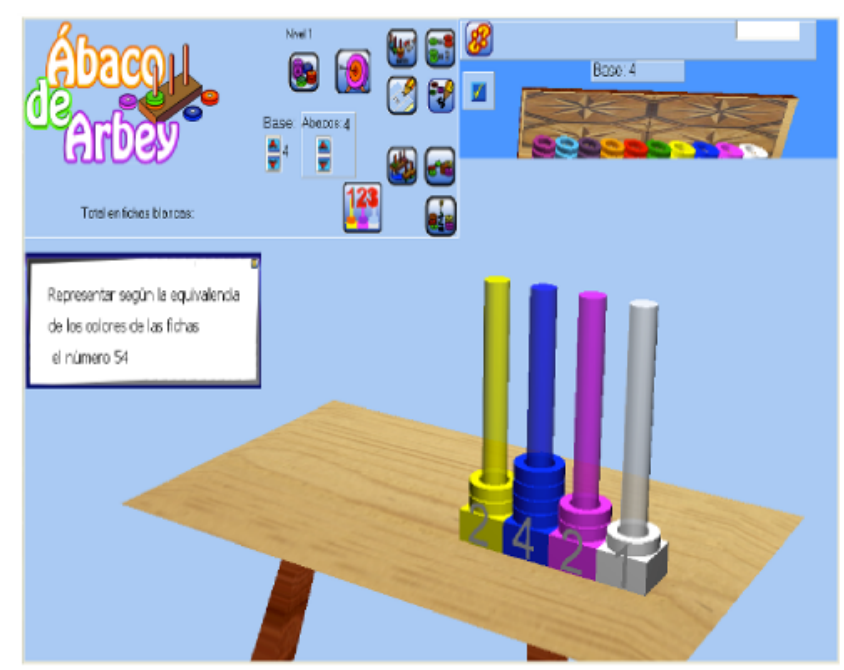

Figura 2. Software Educativo ábaco de Arbey. En esta imagen el software le propone de forma automática el siguiente problema al estudiante: Representar según la equivalencia de los colores (en este caso base 4) el número 54.

El software educativo desarrollado ofrece una serie de actividades interactivas, las cuales se generan de forma automática y complementan el aprendizaje como lo son: el establecer relaciones de orden en diferentes bases, conversión entre bases y problemas de empaquetamiento.

Como ejemplo de algunas de las actividades y preguntas desarrolladas, para lograr el proceso de consolidación conceptual considere el siguiente problema en donde se establece la siguiente relación entre las fichas de la casa de cambio.

1. Analice el esquema del juego de la casa de cambio en base 2 y posteriormente resuelva las preguntas que se le plantean:

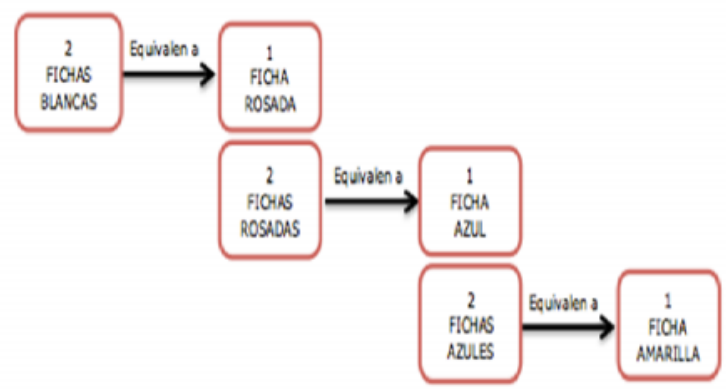

a) ¿A cuántas fichas blancas equivale una ficha amarilla?

b) Iván Camilo tiene 15 fichas blancas y Ana Maria tiene 2 fichas azules. Si los dos cambiaran sus fichas por fichas rosadas iquién tendria más de estas fichas?

Figura 3. Actividad que permite el trabajo de cambio de unidades en la misma base.

\section{METODOLOGÍA DE INVESTIGACIÓN}

La investigación tuvo por objetivo establecer experimentalmente si el método complementado con juegos didácticos influye significativamente en la consolidación del sistema decimal de numeración. Adicionalmente se esperaba diseñar y desarrollar software educativo con juegos didácticos (la casa de cambio y el ábaco abierto), comparar el grado de consolidación del sistema decimal de numeración en dos grupos de estudiantes, en condiciones experimentales diferentes: uno con juegos didácticos computarizados y el otro con juegos didácticos no computarizados, con metodología tradicional, como también describir los aspectos más relevantes del uso de los materiales didácticos tanto computarizados como físicos en la enseñanza de los sistemas de numeración. Por lo tanto la metodología de investigación fue de corte mixto cuasi-experimental y cualitativa. 
El estudio se realizó controlando las siguientes variables intervinientes: género, edad, nivel socio-económico y la escolaridad, en este último caso los estudiantes fueron de un mismo programa académico, y se excluyeron los repitentes. Adicionalmente, para garantizar la homogeneidad académica se les asistió en un cursillo sobre conductas de entrada (pre-requisitos). Como también se consideró homogeneidad en las ayudas educativas, en la intensidad horaria, en el horario de clases, el tiempo dedicado, y en la intervención del profesor, siendo este último el mismo para todos los estudiantes.

Por su parte, el grado de consolidación del sistema decimal de numeración se determinó por el puntaje obtenido por el estudiante ante la aplicación de un instrumento de evaluación el cual nos permitió identificar si:

- Reconoce el valor posicional en diferentes sistemas de representación y realiza conversiones entre unidades de una misma base.

- Realiza conversiones de unidades de mayor orden a unidades de menor orden.

- Calcula la equivalencia en una base determinada, de una ficha de un color dado en fichas blancas y poder realizar el proceso contrario.

- Realiza adiciones y sustracciones de números escritos en una base determinada, utilizando el ábaco abierto.

- Realiza adiciones y sustracciones de números escritos en una base determinada, utilizando procesos simbólicos que se apoyen en los métodos lógicos utilizados en el ábaco abierto.

- Soluciona problemas de transformación de unidades de base 10, multiplicaciones de equivalencia y de correspondencia múltiple.

La población estuvo conformada por 240 estudiantes de grado séptimo de educación básica secundaria de cuatro instituciones educativas públicas del casco urbano del departamento del Quindío en Colombia, con edades entre los 11 y 14 años de edad y perteneciente al casco urbano de cada uno los municipios donde se realizó el estudio.

La muestra se seleccionó de forma aleatoria y se subdividió en dos grupos también con asignación aleatoria para cada institución educativa. Adicionalmente, con el fin de eliminar las diferencias entre los individuos que van a constituir los grupos experimentales y controlar, los factores de selección, mortalidad, histo- ria, maduración y regresión se seleccionó el "diseño de los grupos aleatorios con mediciones antes y después" Campbell y Stanley (1963).

Con respecto al proceso de intervención, este se realizó a través de diez actividades durante un bimestre académico las cuales fueron diseñadas en función de los conceptos y habilidades relacionadas con el grado de consolidación del sistema decimal de numeración. A pesar de trabajar diferentes metodologías en cada uno de los subgrupos, las actividades contenían el mismo material conceptual y las mismas situaciones problema.

\section{RESULTADOS}

A continuación presentamos los resultados del estudio realizado por el grupo de investigadores en relación con el uso del software educativo para la enseñanza del sistema posicional de numeración. Comenzaremos por presentar los resultados del estudio cuasi-experimental el cual consistió básicamente en la prueba $\mathrm{t}$-Student, posteriormente se describen los resultados del estudio descriptivo que se realizó a partir del diario de campo presentando de esta forma los aspectos más significativos durante el proceso de investigación en el aula.

\subsection{Resultados cuantitativos}

La información se obtuvo de la aplicación y evaluación de las actividades que se diseñaron tanto para el tratamiento experimental como para la elaboración del instrumento de medición pre-test - post-test. Para determinar la no existencia de diferencias significativas en los grupos antes de los tratamientos, se aplicó la prueba t -Student a los resultados del pre-test. Para determinar las diferencias significativas en los grupos, debidas a las condiciones experimentales, se aplicó de nuevo la prueba $t$-Student a los resultados del post-test.

\subsubsection{Confiabilidad del instrumento de medición}

Para medir la confiabilidad del instrumento de medición (Test), se utilizó el método de las dos mitades por ser el más adecuado a la naturaleza del instrumento. Este coeficiente se denomina "COEFICIENTE DE CONSISTENCIA" y se calcula por medio de la fórmula de Spearman Brown, ver (Hoyos, 2002) y (Hoyos,2007). Para calcularlo, se aplicó el instrumento a 21 estudiantes de grado octavo de básica secundaria obteniendo una confiabilidad del $97.33 \%$. 
Influencia de software educativo en la consolidación del sistema de numeración. Acosta et al.

\subsubsection{Homogeneidad de los grupos}

Para comprobar la homogeneidad de los grupos juegos didácticos físicos y software educativo, se aplicó la prueba estadística t-Student al Pre-test. Los resultados de los estudiantes de las cuatro instituciones, obtenidos mediante un paquete estadístico se pueden apreciar en la Figura. 4:

\begin{tabular}{lcc}
\hline & $\begin{array}{c}\text { Pretest Grupo } \\
\text { Control Juegos } \\
\text { Didácticos }\end{array}$ & $\begin{array}{c}\text { Pretest Grupo } \\
\text { Experimental } \\
\text { Software }\end{array}$ \\
\hline Recuento & 120 & 120 \\
Promedio & 1,26978 & 1,0553 \\
Desviación Estándar & 1,00294 & 1,08226 \\
Coeficiente de & $78,9851 \%$ & $102,555 \%$ \\
Variación & & \\
Mínimo & 0 & 0 \\
Máximo & 4,26 & 7,14 \\
Rango & 4,26 & 7,14 \\
Sesgo Estandari- & 2,23353 & 8,80533 \\
zado & & \\
Curtosis Estandari- & $-0,986929$ & 16,6185 \\
zada & & \\
\hline
\end{tabular}

Pretest_Grupo_Control

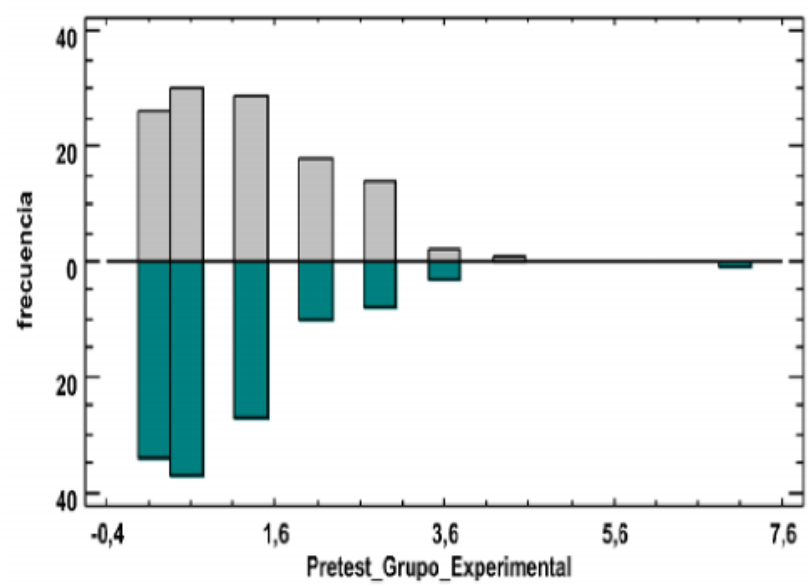

Figura 4. Resultados de la prueba t-Student, para comprobar la homogeneidad de los grupos al inicio de la intervención didáctica.

Utilizando un intervalo de confianza del $95,0 \%$ para la media de Pretest Grupo Juegos Didácticos Físicos: $1,26978+/-0,181289$ [1,08849, 1,45107], un intervalo de confianza del $95,0 \%$ para la media de Pretest Grupo_Software: $1,0553+/-0,195627 \quad[0,859673$, $1,25093]$, y un intervalo de confianza del $95,0 \%$ para la diferencia de medias suponiendo varianzas iguales, se tiene: $0,214483+/-0,26535 \quad[-0,0508668$, $0,479833]$, con $\mathrm{t}=1,59235$ y valor- $\mathrm{P}=0,112634$.
Los resultados obtenidos con el valor de $\mathrm{P}=0,112634$ $>0.05$, en la prueba de análisis de medias de dos muestras son superiores al $\alpha=0.05$ (nivel de significancia) por lo cual no existe diferencias significativas entre los grupos control (juegos manipulativos) y experimental (software educativo) de acuerdo con los resultados obtenidos al aplicar el Pre-test, proporcionando los elementos estadísticos suficientes para afirmar la homogeneidad de los grupos, y proceder a la aplicación de la estrategia didáctica.

\subsubsection{Análisis estadístico posterior al proceso de in- tervención didáctica}

Para el análisis estadístico de los datos, se comparó el pos-test de ambos grupos de los estudiantes de las cuatro instituciones, se procedió a utilizar la prueba estadística t-Student obteniendo los resultados de la Figura. 5:

\begin{tabular}{lcc}
\hline & $\begin{array}{c}\text { Postest Grupo } \\
\text { Control } \\
\text { Juegos Didác- } \\
\text { ticos }\end{array}$ & $\begin{array}{c}\text { Postest Grupo } \\
\text { Experimental } \\
\text { Software }\end{array}$ \\
\hline Recuento & 120 & 120 \\
Promedio & 4,76887 & 4,89954 \\
Desviación Estándar & 2,1421 & 2,48892 \\
Coeficiente de & $44,9184 \%$ & $50,799 \%$ \\
Variación & & \\
Mínimo & 0 & 0,71 \\
Máximo & 9,282 & 10,0 \\
Rango & 9,282 & 9,29 \\
Sesgo Estandari- & 0,128904 & 0,652166 \\
zado & & $-2,21391$ \\
Curtosis Estandari- & $-1,61995$ & \\
zada & & \\
\hline
\end{tabular}

Postest_Grupo_Control

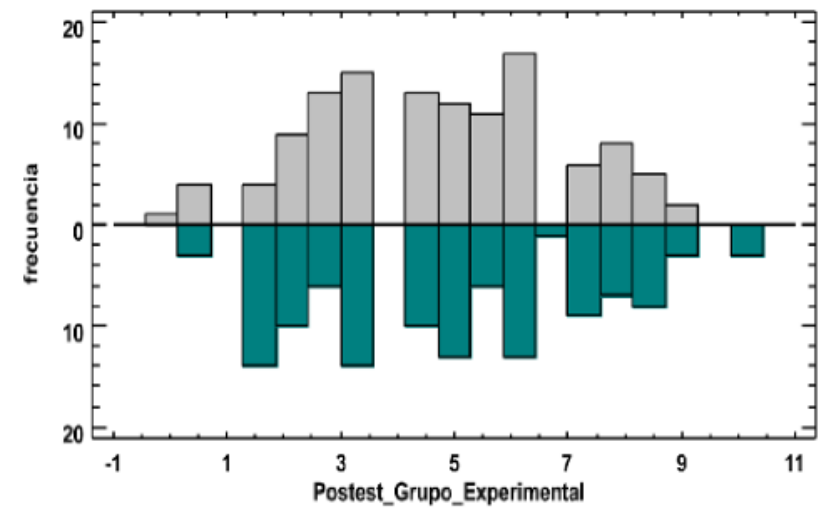

Figura 5. Resultados de la prueba t-Student, después del proceso de intervención didáctica. 
Utilizando un intervalo de confianza del $95,0 \%$ para la media de Postest_Grupo_Juegos Didácticos Físicos: $4,76887+/-0,387201 \quad[4,38167,5,15607]$, un intervalo de confianza del $95,0 \%$ para la media de Postest Grupo_Software: 4,89954 +/- 0,449892 [4,44965, $5,34943]$, y un intervalo de confianza del $95,0 \%$ para la diferencia de medias suponiendo varianzas iguales, se tiene: $-0,130675+/-0,590539 \quad[-0,721214$, $0,459864]$, con $\mathrm{t}=-0,43592$ valor- $\mathrm{P}=0,66329$.

Los resultados obtenidos con el valor de $\mathrm{P}=0.66329$ $>0.05$, en la prueba de análisis de medias son superiores al $\alpha=0.05$ (nivel de significancia) por lo cual se afirma que no existen diferencias significativas entre los grupos juegos didácticos físicos y software de acuerdo con los resultados obtenidos al aplicar el pos-test; aunque se indica que la estrategia didáctica aplicada al grupo que usó el software educativo, no tuvo mayor incidencia en el proceso de aprendizaje, sí tuvo un valor promedio mayor comparado con el grupo control, en donde se usaron los juegos manipulativos.

\subsection{Análisis cualitativo}

Con respecto al seguimiento realizado en el proceso de intervención de cada uno de los grupos se pudo observar que en general los estudiantes no tenían unas bases sólidas en la realización de las cuatro operaciones aritméticas básicas, con especial dificultad en la resta y la división, sin embargo, después de terminar con la secuencia didáctica, se observó una mejora en el manejo de dichas operaciones. Con respecto a la actitud de los estudiantes, se notaron dispuestos a trabajar tanto en la sala de informática como en el aula de clase con los materiales manipulativos. A continuación se transcriben las observaciones del docente en relación con los grupos de materiales manipulativos y material computarizado.

En el grupo que utilizó la metodología de juegos manipulativos, por un lado, se contó con una gran disposición e interés por parte de los estudiantes frente al juego de "La Casa de Cambio", muchos de ellos lograron desarrollar la habilidad de cálculo mental, para resolver los distintos problemas que plantea el juego, sin embargo, cuando se llegó el momento de pasar a la parte simbólica, y resolver los problemas planteados en las guías, los estudiantes entraron en conflicto, pues aunque llegaban a la respuesta correcta, decían no saber cuáles eran esos procesos mentales que les permitía responder de forma co- rrecta, produciendo cierta dificultad para escribir las operaciones necesarias para resolver los problemas propuestos en las guías. Por otro lado, la utilización del Abaco abierto contó con una gran acogida entre los estudiantes, ya que facilitó la comprensión de varios conceptos de los sistemas posicionales, así como la optimización del proceso de la suma y la resta en una base numérica distinta a la base decimal.

En la metodología con Software, los estudiantes se mostraron entusiasmados al saber que aprenderían la temática propuesta, a través del uso de computadores. La experiencia vivida en el colegio muestra en cuanto a la utilización del software, una secuencia lógica con respecto a la apropiación de las reglas de los sistemas de numeración. Por ejemplo, se observó que los estudiantes para lograr una conceptualización en el trabajo con el Ábaco Abierto en el computador, antes debían comprender las reglas del sistema posicional, este se logró gracias a la utilización del juego de la Casa de Cambio, un software sencillo, un tanto mecánico pero útil el cual permite una consolidación del concepto posicional. Al tener este paso de la Casa de Cambio al Abaco, se observa ya una utilización de procesos mentales, algo que no se nota en la Casa de Cambio, además en el Abaco, si hay una restricción de la cantidad de fichas dadas por cada posicionamiento. En conclusión, se debe dar una combinación de varias herramientas para lograr una buena apropiación de un concepto a aprender.

En cuanto a las observaciones hechas durante la evaluación de la prueba se destacan las diversas heurísticas utilizadas por los estudiantes y las dificultades de los mismos para realizar los problemas de conversión entre bases, como es el caso de la siguiente pregunta: utilice el ábaco abierto para calcular la cantidad de fichas de cada color que puedo reclamar con 42 fichas blancas, si se juega en base 3.

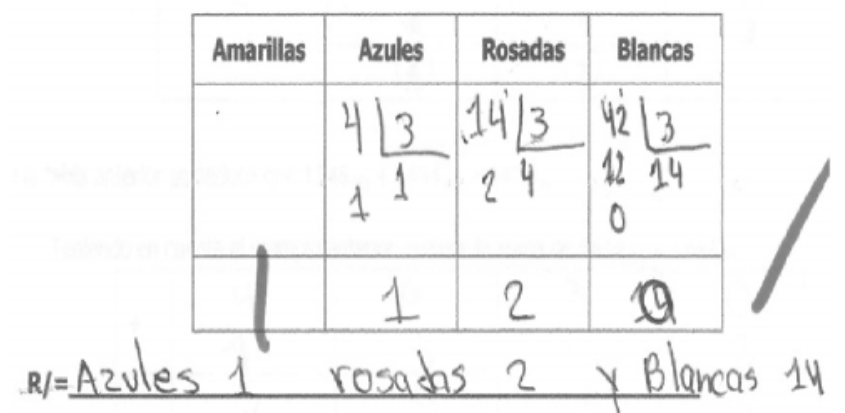

(a). Respuesta incorrecta. 


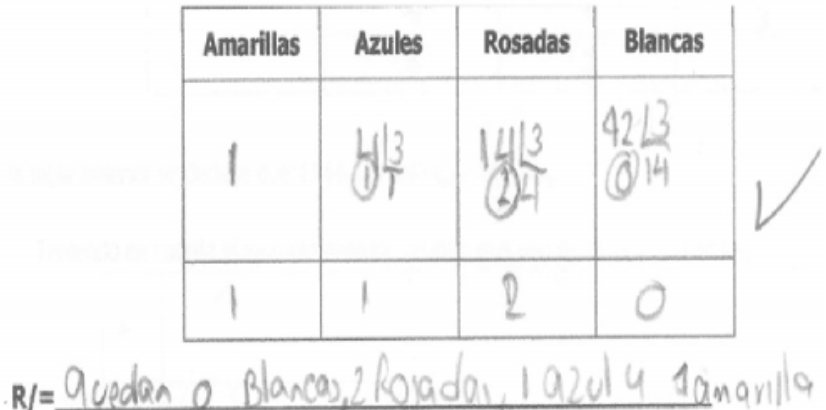

(b) Respuesta correcta.

Figura 6. Respuestas de un par de estudiantes en relación con el problema de conversión de base 10 a base 3 . Pregunta: Utilice el ábaco abierto para calcular la cantidad de fichas de cada color que puedo reclamar con 42 fichas blancas, si se juega en base 3. (a) Respuesta incorrecta, (b) Respuesta correcta.

Como se aprecia en la Figura.6a el estudiante al seguir un procedimiento memorístico, utiliza simplemente el cociente de la división como respuesta y no reconoce que al agrupar de a tres las 42 fichas blancas no sobra alguna y por tanto no deben quedar fichas blancas, esto es, no usa el residuo de la división para significar el número de fichas blancas restantes. Entre otras dificultades en este tipo de problemas se destaca las dificultades para efectuar las divisiones. Sin embargo, la respuesta correcta Figura.6b fue expresada por varios estudiantes.

Por su parte, el proceso inverso requiere de la evaluación de un polinomio como se establece en la siguiente pregunta: Si se sabe que el ganador del Juego de la Casa de cambio en base 3 termina con 2 fichas amarillas, 1 azul, 2 rosadas y 1 blanca. A cuántas fichas blancas equivalen las fichas obtenidas por el ganador del juego?. En esta pregunta se destaca que algunos estudiantes no reconocen el valor posicional, esto es, no establecen la relación entre cada uno de los colores como se aprecia en la Figura 7a el estudiante simplemente multiplica por 3 cada una de las fichas de cada color y luego las suma. Por otro lado, la Fig. $7 \mathrm{~b}$ nos muestra como el estudiante por medio de las relaciones existentes entre cada uno de los colores le permite evaluar el polinomio:

$$
2 \cdot 3^{3}+3^{2}+2 \cdot 3+1
$$

en la forma anidada,

$$
((2 \cdot 3+1) \cdot 3+2) \cdot 3+1
$$

la cual corresponde a una forma computacionalmente eficiente de evaluar un polinomio, ver (Knuth,1981).

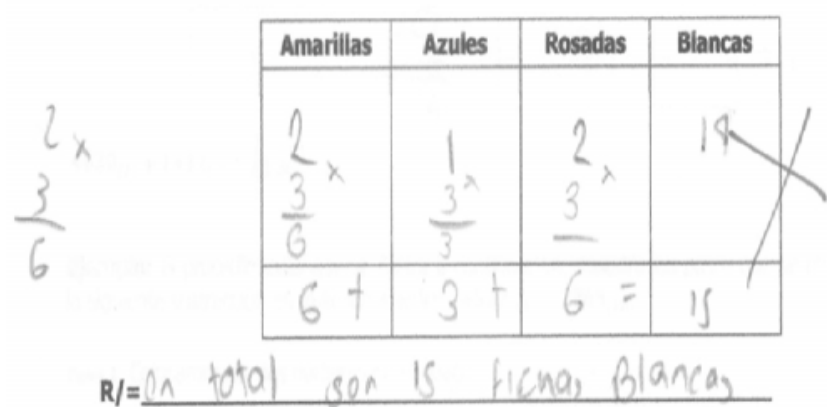

(a). Respuesta incorrecta.

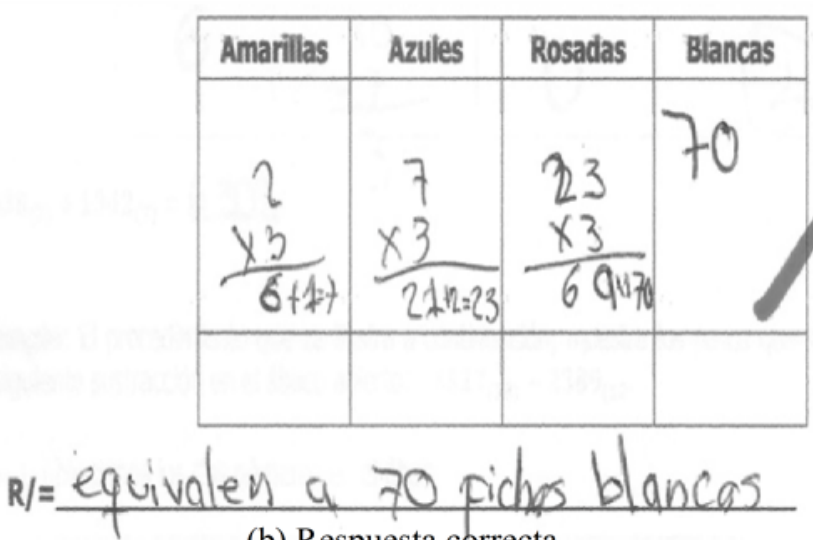

(b) Respuesta correcta.

Figura 7. Respuestas de un par de estudiantes en relación con el problema de conversión de base 3 a base 10. Pregunta: Si se sabe que el ganador del juego de la casa de cambio en base 3 termina con 2 fichas amarillas, 1 azul, 2 rosadas y 1 blanca. A cuántas fichas blancas equivalen las fichas obtenidas por el ganador del juego? (a) Respuesta incorrecta, (b) Respuesta correcta.

\section{DISCUSIÓN Y CONCLUSIONES}

En este trabajo se presentaron los resultados de una investigación en relación con el uso de una propuesta didáctica apoyada con material didáctico computarizado para la consolidación del sistema de numeración posicional en base 10 a partir de la lógica de los sistemas posicionales. Considerando el planteamiento del problema y en lo atinente a los objetivos de investigación, al análisis cuantitativo y a la descripción cualitativa del seguimiento a las actividades es posible concluir que desde el punto de vista cuantitativo, la prueba $\mathrm{t}$-Student aplicada a los resultados del pretest confirmó la hipótesis de que no existe diferencia académica significativa entre estos grupos. Esto indica que no hay diferencias significativas entre las varianzas de los grupos control y experimental, proporcionando los elementos estadísticos suficientes para afirmar la homogeneidad de los grupos, para la aplicación de la estrategia didáctica. De forma similar, la prueba $\mathrm{t}$-Student aplicada al post-test indica que no 
hay diferencias significativas entre las varianzas de los grupos control y experimental, esto indica que la estrategia didáctica aplicada al grupo experimental no tuvo mayor incidencia en el proceso de aprendizaje, aunque sí tuvo un valor promedio mayor en el grupo experimental que en el grupo control.

Este estudio permitió a los investigadores evidenciar de forma cuantitativa y cualitativa que tanto los grupos experimentales como en el grupo control se lograron avances significativos debido a cada uno de los tratamientos. Los materiales manipulativos y computarizados son una importante herramienta didáctica en la enseñanza y en la consolidación de los sistemas posicionales de numeración. El software diseñado por el grupo, al generar de forma automática los problemas, permitió a los estudiantes trabajar de forma autónoma e independiente, allí los estudiantes más adelantados tuvieron la oportunidad de poner a prueba su conocimiento en relación con el sistema de numeración posicional. En este sentido, la metodología con software facilita la labor del docente y permite que los estudiantes junto con la motivación intrínseca que produce el computador resuelvan más problemas en menos tiempo, permitiendo de esta forma consolidar y reforzar su aprendizaje.

Indirectamente, por lo menos, estos resultados permiten confirmar algunos puntos de vista o resultados de otras investigaciones mencionadas en otras secciones de este trabajo. En efecto, la utilización del computador como elemento didáctico es una necesidad porque permite adoptar nuevas formas metodológicas en la enseñanza de las matemáticas. La motivación que las mismas generan en los estudiantes es un valor agregado que el docente definitivamente debe explorar. Se espera que las experiencias de este trabajo permitirán el desarrollo y formulación de nuevos proyectos de investigación y de formación docente en el área de inclusión de tecnología al currículo de las matemáticas a nivel regional y nacional.

Agradecimientos: Esta investigación fue financiada por el Ministerio de Educación Nacional de Colombia a través de Colciencias con código 1113-578-36096 correspondiente al contrato 258 de 2013, como también por la Universidad del Quindío y la gobernación del Quindío, Colombia.

\section{BIBLIOGRAFÍA}

1. Baroody, A. (2000). El pensamiento matemático de los niños, Editorial: Visor Dis. S.A. Madrid España.

2. Cadavid, G. S. (2013). Enseñanza del valor posicional en el sistema de numeración decimal para niños de escuela básica usando las nuevas tecnologías. Tesis de Maestría, Universidad Nacional de Colombia. Facultad de Ciencias. Sede Medellín. Colombia.

3. Campbell, D.T. \& Stanley, J.C. (1963). Experimental and quasi-experimental designs for research, Houghton Mifflin Company Boston.

4. Castaño, J., Negret, J. C. \& Robledo, A. M. (1990). Un marco para la comprensión de la construcción del sistema decimal de numeración en los niños. Reporte Técnico. Pontificia Universidad Javeriana. Facultad de Psicología. Santafé de Bogotá.

5. Cid, E., Godino, J.D. \& Batanero, C. (2003). Sistemas numéricos y su didáctica para maestros, ReproDigital. Facultad de Ciencias. Avda. Fuente nueva 18071 Granada.

6. Galvis, A. (1994). Ingeniería de software educativo, Ediciones Uniandes. Colombia.

7. Garay, H. (2008). Sugerencias para una integración curricular de la tecnología educativa, Quehacer Educativo 88.

8. Guarín, S. E. (2012). Enseñanza y aprendizaje del sistema de numeración decimal, a partir de las Tics y la educación artística como estrategia de aprendizaje significativo, en el grado segundo de Educación Básica Primaria de la Institución Educativa Vallejuelos. Municipio de Medellín año 2012 (tesis de posgrado). Universidad Nacional de Colombia, Medellín, Colombia.

9. Hoyos Salcedo, E. A. (2012). Representación de objetos tridimensionales utilizando multicubos, VIII Festival Internacional de Matemática Universidad Nacional.

10. Hoyos Salcedo, E. A., Grisales, A. \& Cerón, M. A. (2007). Estrategia de intervención pedagógica con juegos didácticos computarizados que contribuyan a la consolidación del esquema multiplicativo sim- 
ple, Revista de Investigaciones de la Universidad del Quindío ISSN: 1794-631X 17, no. 1, 149-158.

11. Hoyos Salcedo, E. A., Grisales, A. \& Cerón, M. A. (2002). Estudio experimental del uso de un geoplano computarizado en la enseñanza de la geometría euclidiana del cuarto nivel de educación básica, Revista De Investigaciones de la Universidad del Quindío ISSN: 1794-631X 4, no. 11, 79-87.

12. Knuth, D.E. (1981). The art of computer programming, Addison-Wesley Publishing Company.

13. Lengnink, K. \& Schlimm, D. (2010). Learning and understanding numeral systems: Semantic aspects of number representacions from an educational perspective, Philosophy of Mathematics: Sociological Aspects and Mathematical Practice. College Publications. London.

14. Lerner, D. \& Sadovsky, P. (1997). Didáctica de matemáticas, ch. El sistema de numeración: un problema didáctico, Paidós Educador.

15. MEN, (2003). Tecnologías computacionales en el currículo de matemáticas, Cooperativa Editorial Magisterio Santafé de Bogotá.

16. MEN, (1998). Ministerio de educación nacional: Matemáticas serie lineamientos curriculares, Cooperativa Editorial Magisterio Santafé de Bogotá.

17. MEN, (1999). Nuevas tecnologías y currículo de matemáticas, Cooperativa Editorial Magisterio Santafé de Bogotá.

18. Ramírez Castro, O. A. (2011). Sistema de numeración posicional como estructura matemática: Una experiencia didáctica con estudiantes de grado sexto. Tesis de Maestría. Maestría en enseñanza de las ciencias exactas y naturales. Universidad Nacional de Colombia.

19. Salazar, C. Y, \& Vivas Y. A. (2013). Enseñanza del sistema de numeración decimal a través de la integración de material manipulativo, (tesis de pregrado). Universidad del Valle, Santiago de Cali, Colombia.

20. Terigi, F. \& Wolman, S. (2007), Sistema de numeración: Consideraciones acerca de su enseñanza, Revista Iberoamericana de Educación, no. 43, 59-83.

21. (2011). Rights Reserved Ventura Educational Systems, Tools for active teaching and active learning hand on math base ten blocks in structure guide.

22. Zhang, J. \& Norman, D. A. (1995). A representational analysis of numeration systems, Cognition, no. $57,271-295$. 